Rev. High Pressure Sci. Technol., Vol. 7 (1998) 867 869

\title{
Temperature Measurements during Shock Compaction of Powders
}

\author{
E.P. Carton*, , M. Stuivinga*, H.J. Verbeek*, and P.J.J.M. van der Put ${ }^{+}$ \\ *TNO Prins Maurits Laboratory, P.O. Box 45, 2280 AA, Rijswijk, The Netherlands \\ +Delft University of Technology, Laboratory for Applied Inorganic Chemistry, P.O. Box 5045, 2600 GA, Delft, The Netherlands
}

\begin{abstract}
The measurement of the temperature history of a powder during the shock compaction process requires a very fast measuring principle. Since the response time of normal thermocouples is too slow for the extremely fast temperature changes occurring in the shock compression of powders, here thermocouples formed by the powder to be compacted are used. The temperature measurements are performed in the cylindrical configuration. Copper and constantan powders are used in the development of the measuring technique. This resulted in measurements of the temperature history in these metallic materials. To be able to investigate whether the temperature history during the shock compaction of ceramics differs from that of metals, two ceramic materials $\mathrm{TiB}_{2}$ and WC) were selected to be used as a powder thermocouple for future work. [powder, shock compaction, heterogeneous temperature distribution, thermocouple, ceramic]
\end{abstract}

\section{Introduction}

The heterogeneous temperature distribution occurring in the shock compaction of powders is believed to play an important role in the consolidation process. Temperature measurements can be a tool to determine the rise time of the shock front and the energy dissipation which are important to understand the bonding mechanisms between the particles. Within the shock front (having a rise time less then one microsecond in powders) most compaction, mass acceleration and energy dissipation occurs. The increase in internal energy $(\Delta \mathrm{E})$ of the powder material in the shock wave can be approximated by $\Delta E=1 / 2 \mathrm{P}$ $\Delta \mathrm{V}$, in which $\mathrm{P}$ is the shock wave pressure, and $\Delta \mathrm{V}$ the accompanying change in specific volume of the powder. In shock waves propagating in powders, the increase in internal energy is rather large compared to solids, due to the large irreversible volume change $(\Delta V)$ during its compaction. Most of this energy is dissipated at the surface of the powder particles and results in local heating of these areas. This creates a very heterogeneous temperature distribution in the powder compact, which homogenizes fast due to heat conduction toward the interior of the particles. This is schematically shown in Fig.1, together with the corresponding pressure profile. Measurement of such fast temperature variations have been performed for metallic powders in a plane wave geometry, for example by Schwarz et al. [1] and Mutz et al. [2].

In this paper we report similar measurements using the cylindrical geometry for compaction.

\section{Thermocouple principle}

The potential difference $\left(U_{E M F}\right)$ between two points within an electric conducting material $\mathrm{A}$ is, within a certain temperature range, a lineair function of the difference in temperature $\left(T_{1}-T_{0}\right)$ between these points. The proportionality constant, which is a property of the material, is called the Seebeck-coefficient, $\mathrm{S}_{\mathrm{A}}: \mathrm{U}_{\mathrm{EMF}}=\mathrm{S}_{\mathrm{A}}\left(\mathrm{T}_{1}-\mathrm{T}_{0}\right)$.

A second material with a different Seebeck-coefficient $\left(S_{B}\right)$ connected at the hot side (hot junction) is needed in order to measure a potential difference between the two materials at the cold side with temperature $\mathrm{T}_{0}$ (the cold junction):

$$
\Delta \mathrm{U}_{\mathrm{EMF}}=\left(\mathrm{S}_{\mathrm{A}}-\mathrm{S}_{\mathrm{B}}\right)\left(\mathrm{T}_{1}-\mathrm{T}_{0}\right)
$$

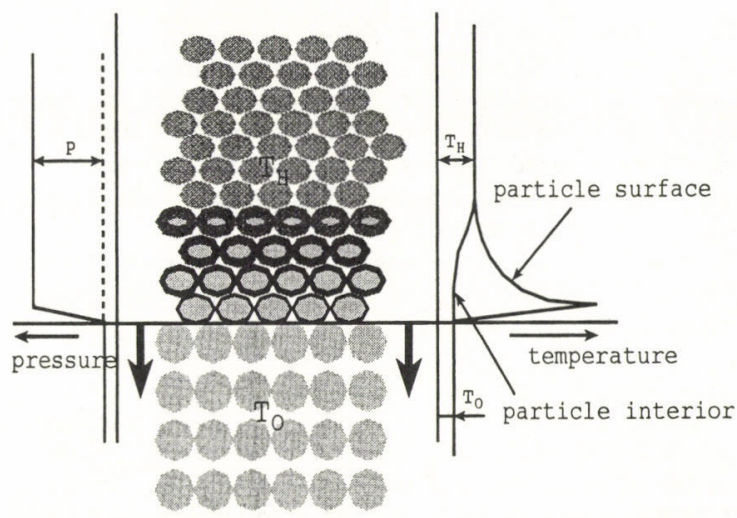

Fig. 1. Temperature distribution and profile during shock compaction of a powder.
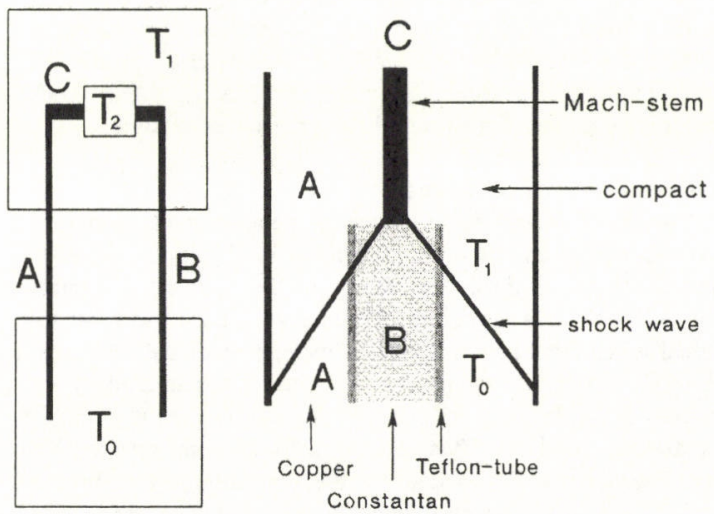

Fig. 2. Schematic representation of a thermocouple (left), and the powder thermocouple in the cylindrical configuration (right). 


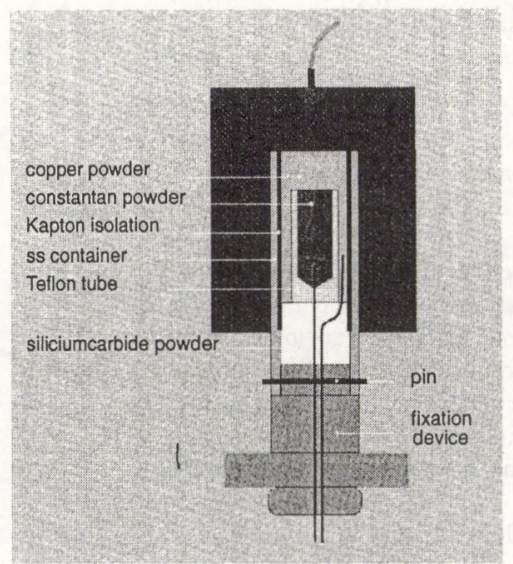

Fig. 3. Experimental set-up for measuring the temperature profile in powders in the cylindrical configuration.

The difference in relative Seebeck-coefficients $\left(\mathrm{S}_{\mathrm{A}}-\mathrm{S}_{\mathrm{B}}\right)$ determines the thermoelectric power of the thermocouple. Usually, a thermocouple is inserted in the medium in which the temperature has to be measured. The two wires of material $\mathrm{A}$ and $\mathrm{B}$ are on one side electrically connected, this may be done by a third material $\mathrm{C}$ as long as it does not incorporate a net temperature gradient [3], see Fig. 2.

This couple then is heated by the medium via heat conduction. Even the small response time of very thin thermocouples is not enough to measure the extremely fast heating of the particle surface occurring in the shock compaction of a powder. However, when the shock compacted powder itself forms the thermocouple (powders of material A and B) it is possible to measure the extremely fast temperature changes, since they do not depend on the process of heat conduction from the particles to the temperature sensor. Ideally, the powders which form the thermocouple should have the same shock impedance. Then the shock wave will not be disturbed when it passes the powder interface. Also the temperatures generated in the powders behind the shock front should have the same value. Both the impedance and the temperature depend strongly on the initial density of the powder. Although this makes it possible to compensate for differences in intrinsic material properties, preferably materials are chosen which have the same shock impedance in the solid phase, like copper and constantan.

\section{Experimental arrangement}

The experimental set-up for the temperature measurement in the cylindrical configuration is shown in Fig. 3. In order to obtain the full temperature history of the powder, a fixation device for the powder container was developed. It prevents the signal wires from breaking due to movement of the container. Without a fixation system, the container is accelerated by the forces generated on it by the detonation or its rapidly expanding products. Just above the fixation system, the container is not surrounded by the explosive layer. In this region the shock wave will attenuate, reducing the forces acting on the fixation device and the simple pin-hole bonding between the device and the powder container. After the experiment the

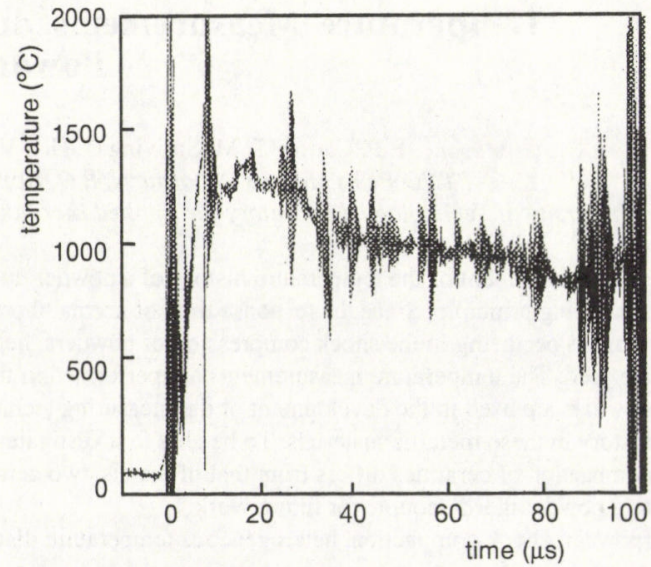

Fig. 4. Experimental result of a temperature measurement with copper-constantan powders

container can easily be removed and the fixation device can be used many times.

During the development of the dynamic temperature measurement, several sources of noise have been reduced to an acceptable level. The main source of electromagnetic noise appeared to be the PVC-tube that was used as the explosive container. After replacing this material with card-board only the electromagnetic noise due to the electric detonator and the ionization pin, which triggers the oscilloscope, was still present. By using a wire explosive on which the detonator and the ionization pin were applied, their noise could be separated in time from the thermocouple signal. Between the explosive and the explosive wire a booster charge had to be applied, since the wire explosive could not directly initiate the explosive layer.

In the experiments described here, the interface of the two powders making up the thermocouple is flat and normal to the detonation direction. This means that the oblique shock wave generated in the powder cannot cross the entire interface instantaneously. However, when the angle of the shock wave towards the cylinder axis is known, for example by measuring it from a flash $\mathrm{x}$-ray photograph [4], the interface can easily be shaped as a cone with the right cone angle. This can be accomplished by using a die with the cone shaped hole in the bottom during the static precompaction of the powder to its starting density. This shaped interface will be crossed instantaneously by the shock front, which is required to be able to measure the rise time of the shock front and the accompanying temperatures.

\section{Experimental results and discussion}

The first experiments were performed with copper and constantan powders with a normal particle size distribution of 50 and $150 \mu \mathrm{m}$, respectively. The starting density of both powders was $55 \%$ of theoretical maximum density (TMD). After shock compaction with AMPA (an AN based explosive with a detonation velocity of $3.6 \mathrm{~km} / \mathrm{s}$ ), the density had increased to 97 \%TMD and a hole in the center of the compact had been formed. Apparently a Mach reflection (Mach stem) had occurred here. 


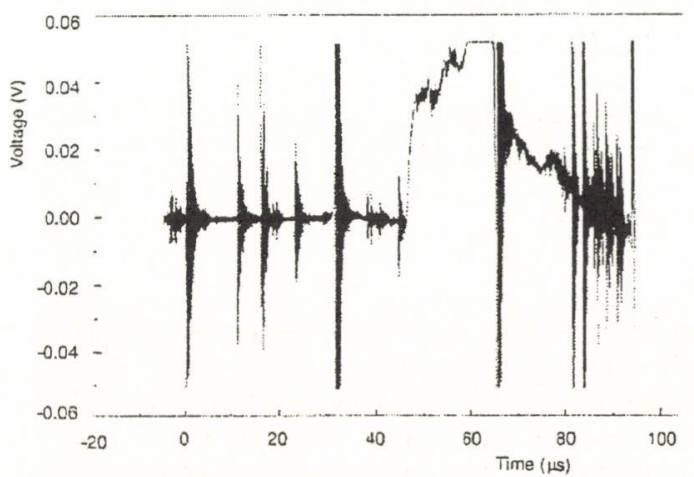

Fig. 5. Experimental result of a temperature measurement with bimodal copper-constantan powders with higher starting densities.

In the Mach stem, indicated as conducting material $\mathrm{C}$ in Fig. 2, the material melts and attains a high axial velocity. In Fig. 4 the measured temperature profile is shown. Here the thermovoltage has been converted to temperature using the results of a calibration with copper and constantan wires in an oven. At $\mathrm{t}=0$ the ionization pin triggers the oscilloscope. Around 10 microseconds after arrival of the shock wave at the copperconstantan interface the melting point of constantan $\left(1388^{\circ} \mathrm{C}\right)$ is measured. Thereafter the temperature reduces gradually to the melting point of copper at normal pressure $\left(1083^{\circ} \mathrm{C}\right)$. Although this experiment is not a good example of the heterogeneous temperature distribution within the powder, however, it shows that the measured thermovoltage corresponds well with temperatures occurring during the compaction process.

The Mach stem can be prevented by using a higher starting density of the powder materials [5]. This can be obtained by using a bi- or trimodal particle size distribution.

Here, bimodal particle size distributions of both the copper (50 and $150 \mu \mathrm{m})$ and constantan $(150 \mu \mathrm{m}$, mixed with $20 \mathrm{vol} \%$ alumina particle of $50 \mu \mathrm{m}$ ) were used. This increased the starting densities to $73 \% \mathrm{TMD}$ and $63 \% \mathrm{TMD}$, respectively. The temperature measurement is shown in Fig. 5. The signal has been recorded from the moment of triggering $(t=0)$ for a period of $100 \mu \mathrm{s}$. From the distance between the ionization pin and the copper-constantan interface and the detonation velocities of the explosives used, a lower bound for the expected starting time of the temperature increase has been calculated to be $40 \mu \mathrm{s}$. This is in reasonable agreement with the measured starting time of the temperature increase at $47 \mu \mathrm{s}$. At this moment the signal jumps in about $1 \mu \mathrm{s}$ to $37 \mathrm{mV}$, representing a temperature of about $650{ }^{\circ} \mathrm{C}$. The second temperature increase could be due to the reflected shock wave at the cylinder axis, increasing the pressure for the second time. Although the pressure increase in the second shock wave can be considerable, main compaction $(\Delta \mathrm{V})$ and hence temperature increase is obtained in the initial shock wave [6].
The temperature stayed well below the melting point of the materials.A cross-section of the compact after the experiment showed a homogeneous density distribution, without indications that a Mach stem had occurred. Since the compaction behavior of (brittle) ceramic powders is different from metallic powders, further research is concentrating on applying ceramic powders to form the thermocouple. Although it proved to be hard to get data concerning the thermoelectric properties of ceramic materials, a selection was made on the basis of Seebeck-coefficient and electric conductivity. The thermoelectric power of the couple should be high in order to get measurable signals, so the materials that make up the couple must have a large difference in the value of the Seebeckcoefficient. The electric conductivity of most ceramics is rather poor compared to metals, and for a powder the conductivity is reduced at least one order of magnitude with respect to the value for the dense solid material. $\mathrm{TiB}_{2}$ and $\mathrm{WC}$ have been selected here, since these ceramics posses electric conductivities of the same order of magnitude as metals, and together have a relative Seebeck-coefficient of $20 \mu \mathrm{V} / \mathrm{K}$ [7].

\section{Conclusions}

A fixation device has been developed enabling electrical measurements to be performed during and right after shock compaction of powders in the cylindrical configuration.

The temperature history of metallic powders during their shock compaction has been measured. A good relation between the measured temperatures and the physically occurring processes during the shock compaction was obtained. The latter were concluded from microscopic observations of the preserved samples.

$\mathrm{TiB}_{2}$ and WC have been selected as materials to construct a ceramic thermocouple on the basis of their Seebeck-coefficients and electrical conductivity values.

\section{References}

[1] R.B. Schwarz, P. Kasiraj, and T. Vreeland, jr., Proceedings of the Int. Conf. on: Metall. Appl. of Shock-Wave and HighStrain-Rate Phenomena, edited by L.E. Murr, K.P. Staudhammer, and M.A. Meyers, 313 (1986).

[2] A.H. Mutz, and T. Vreeland, jr., J. Appl. Phys. 73, 4862 (1993).

[3] D.D. Pollock, Chapter 4 in Thermocouples Theory and Properties, CRC Press, Boston, 1987.

[4] E.P. Carton, H.J. Verbeek, M. Stuivinga, and J. Schoonman, J. Appl. Phys. 813038 (1997).

[5] E.P. Carton, M. Stuivinga, H.J Verbeek, Presentation at the APS Conf.: Shock Compression of Condensed Matter 1997, "Crack Prevention in Shock Compression of Powders", 27 July-1 August, Amherst, MA, USA ,1997.

[6] R.A. Graham, Chapter 4 in High Pressure Explosive Processing of Ceramics, R.A. Graham, and A.B. Sawaoka eds., Trans Tech Publications, Switzerland, 1987.

[7] Yu. M. Goryachev, and T.M. Yarmola, Translation of the Fourth Conf. on Ceramics for Electronics, 1-12, May 1971, by Leo Kanner Associetes, Redwood City, CA, USA (1974). 